\title{
Organisationsinternes Coaching
}

\author{
Astrid Schreyögg
}

Bei Durchsicht der einschlägigen Literatur, etwa in der Sammlung von Christopher Rauen, fällt auf, dass die meisten Autoren nur Coaching durch externe Berater beschreiben. Internes Coaching erscheint eher als vernachlässigbares Randphänomen, das für die Gesamtdebatte kaum relevant sei. In der Praxis zeichnet sich allerdings derzeit ein gegenläufiger Trend ab. Im Zuge der „Veralltäglichung von Coaching“ als fast schon selbstverständlichem Bestandteil strategischer Personalentwicklung - wie etwa in der Automobilindustrie oder neuerdings auch in Bundesbehörden - etablieren immer mehr Organisationen nicht nur externe, sondern auch interne Coaching-Pools. Dabei sind die externen meistens fürs Topmanagement zuständig und die internen fürs untere und fürs Mittelmanagement. Auch in einschlägigen Ausbildungszusammenhängen fällt auf, dass in der letzten Zeit viele Personen mit unterschiedlichen akademischen Grundausbildungen, besonders oft als Psychologen, Pädagogen oder Betriebswirte, die interne Coaches werden wollen, die Ausbildungsinstitute für Coaching bevölkern.

Über die Tatsache, dass sich die ,internen“ bislang so wenig in Publikationen geäußert haben, lässt sich nur spekulieren. Ein Grund besteht vielleicht darin, dass sie sich scheuen, Interna etwa im Sinne von Falldarstellungen aus ihren Systemen zu veröffentlichen. Gelegentlich scheuen sie auch eigens eingeforderte Reviewprozesse durch vorgesetzte Instanzen als unangenehme Kontrolle der eigenen Arbeit. Wahrscheinlich verspüren interne Coaches auch weniger Druck zum Selbstmarketing als externe Berater, denn ihre Klientel ist ihnen ja doch garantiert. Wie dem auch sei, zur Fortentwicklung des Formats Coaching ist es aber doch lohnend, die Debatte ums organisationsinterne Coaching genauer zu beobachten und voranzubringen. Aus diesem Grund widmet auch der Deutsche Bundesverband Coaching (DBVC) im dritten (noch nicht veröffentlichten) Teil seines Kompendiums dem internen Coaching ein eigenes Kapitel.

OSC hat in den letzten Jahren auch immer wieder vereinzelte Beiträge zum internen Coaching publiziert, es fristete aber auch hier eher ein Dasein als Randphänomen. Dann eröffneten aber Stefan Stenzel im Heft 2/2010 und Gabriele Bollhöfer im Heft 1/2011

Online publiziert: 29.04 .2011

(C) VS Verlag für Sozialwissenschaften 2011

Dr. A. Schreyögg $(\bowtie)$

Breisgauer Str. 29, 14129 Berlin, Deutschland

E-Mail: info@schreyoegg.de 
eine ausführliche Debatte zum Thema, indem unterschiedliche Formen des internen Coachings dargestellt und diskutiert werden; und Annelie Eichhorn präsentierte einen Typus des internen Coachings im Universitätsklinikum in Frankfurt. Mit dem vorliegenden Heft wollen wir uns nun in vier Hauptbeiträgen, die allesamt von internen Coaches verfasst wurden, noch umfassender mit dem internen Coaching und seinen Besonderheiten beschäftigen.

Im ersten Aufsatz untersucht Elsbeth Trzaska die Bedeutung von Vertrauen im internen Coaching. Aufgrund der Tatsache, dass hier Coaching oft von vorgesetzten Instanzen verordnet wird, stellt sich das Vertrauensthema in besonders dringlicher Weise. Im Gegensatz zu traditionellen Paradigmen über Vertrauen zwischen Berater und Klient zeigt die Autorin, dass beim internen Coaching immer auch das Vertrauen gegenüber der Organisation relevant ist. Denn der Coach fungiert ja dem Klienten gegenüber nicht nur als je einmaliger Mensch, sondern jetzt auch als Agent der gemeinsamen Organisation. So wirken auch alle organisationskulturellen Muster in den Aufbau der Beratungsbeziehung hinein. Der zweite Fachbeitrag von Andreas Koschuth ist „ein Plädoyer für Coaching vor dem Karrieresprung als fest installiertes Angebot in Organisationen“. Dieser Autor thematisiert zunächst unterschiedliche Konfliktpotenziale beim Einstieg in eine neue Führungsposition. In vielen Systemen, insbesondere der öffentlichen Verwaltung, sei es üblich, dass Menschen ihr gesamtes Berufsleben in ein- und derselben Organisation, aber in unterschiedlichen Funktionen und Hierarchie-Ebenen verbringen. Um ihnen aber nun diese Karrierewege zu erleichtern, sei es sinnvoll, sie auch bei all ihren Karrieresprüngen durch Coaching als selbstverständliches Angebot zu begleiten. Ludger Jakobuß beschreibt eine andere wichtige Funktion von organisationsinternem Coaching, nämlich die Beförderung von Wissenstransfer. Der Autor hat im Rahmen von Tandem-Coaching in einem Großunternehmen dafür gesorgt, dass das Wissen von ausscheidenden Führungskräften an ihre Nachfolger möglichst umfassend und möglichst nahtlos weitergegeben werden konnte. Im vierten Fachbeitrag befasst sich Thorsten Esse schließlich mit den ,strukturell bedingten Herausforderungen des internen Coachings“" und den Möglichkeiten, ihnen zu begegnen. Der Autor fächert zunächst einige Konfliktfelder auf, um sodann zu zeigen, wie insbesondere in der Phase der Kontraktbildung diese Konflikte bearbeitet werden können. Er zeigt darüber hinaus, wie gerade durch die organisatorische Anbindung des internen Coachingangebots die antizipierten Konflikte entschärft werden können.

Die beiden Praxisberichte thematisieren je unterschiedlich innovative Coachingaktivitäten. Im ersten Beitrag berichten Arthur Drexler, Andreas Wieser und Christoph Netzer Effekte von Life-Coaching in einem exklusiven Gesundheitszentrum in den Tiroler Bergen. Philipp Wascher und Thomas Jäger dagegen versuchen zu zeigen, wie sich Coaching als Maßnahme der Personalentwicklung auch in einem Handwerksbetrieb bewähren kann. Im Diskurs schließlich beschäftigt uns Ferdinand Buer mit dem Phänomen „Organisieren“. Anhand von fünf Neuerscheinungen zum Thema fragt er: „Was geschieht da und wie kann man es beeinflussen?" 